\title{
Young patients with movement disorders
}

\author{
UK Ranawaka, AAPT Chang and JC Wijesekera
}

(Index words: Neuro-acanthocytosis, paroxysmal dyskinesias)

\section{Introduction}

Unlike in old people in whom Parkinsonism and related akinetic-rigid syndromes predominate, movement disorders in young adults constitute a diagnostic and therapeutic challenge. Rheumatic chorea and Wilson disease are among the best known, but other disorders are relatively uncommon and may cause diagnostic difficulty. We report two young adults with unusual movement disorders.

\section{Case 1}

A 27-year old man presented with a complaint of 'difficulty in walking'. He had stiffness in the left upper and lower limb on walking, motivating him to sit down and rest after walking some distance. Prolonged grip of an object produced stiffness in his fingers. He also had slurring of speech, curiously also on walking. He was asymptomatic at rest. Distal arterial pulses were normal and there was no blood pressure gradient in the limbs. Duplex scanning of the carotid, vertebral and leg vessels, and myelography were normal. Neurological examination at rest was normal. On walking a short distance, he developed dystonia involving the left upper and lower limbs, with dysarthria. Magnetic resonance imaging (MRI) of the brain and cervical cord, nerve conduction testing and electromyography, and serum creatine kinase levels were normal. A diagnosis of paroxysmal exerciseinduced dystonia was made. He improved with clonazepam and remains reasonably symptom free.

\section{Case 2}

A 33-year old housewife presented with progressive difficulty in walking, abnormal involuntary movements and generalised seizures. She also had episodes of slurring of speech and difficulty in swallowing. Examination showed choreo-athetoid movements in the limbs, pronounced dyskinesias involving the mouth, lips and tongue, ataxia and cognitive impairment. Electroencephalogram showed features of generalised epilepsy. The blood picture was suggestive of neuro-acanthocytosis, with an acanthocyte count of $9 \%$. Neurophysiological studies revealed axonal sensory polyneuropathy. Blood lipid profile and computed tomography scan of the brain were normal. Seizures were well controlled with carbamazepine, but involuntary movements responded poorly to symptomatic treatment.

Both patients were screened for Wilson disease and vasculitis with negative results. Neither had a relevant family history or drug history.

\section{Discussion}

Paroxysmal dyskinesias (previously termed paroxysmal choreo-athetosis and dystonia, now grouped together) are intriguing disorders. They are divided into paroxysmal kinesigenic dyskinesia (PKD, induced by sudden movement), paroxysmal non-kinesigenic dyskinesia (PNKD, no specific triggers), paroxysmal exercise induced dyskinesias (PED) and paroxysmal hypnogenic dyskinesia (during non-REM sleep) [1,2]. PED may be sporadic or familial (autosomal dominant) $[1,3]$. Sporadic cases usually present with dystonia involving lower limbs [1]. Dystonic movements are characteristically precipitated by exercise, may be unilateral, and last 5 to $30 \mathrm{~min}$. Frequency may vary from a few attacks in a month to several in a day $[1,3]$. Dysarthria is a common accompaniment. Passive movements or sensory stimulation of the limbs may also act as triggers $[1,3]$. Sensory symptoms in affected limbs, postural tremor and migraine may be associated [1]. Some patients have prodromal symptoms and post-ictal phenomena [1], and exclusion of seizures is important. EEG and neuroimaging are normal. Treatment is usually with antiepileptics, acetazolamide or clonazepam, but the response is not always good [3].

Neuro-acanthocytosis is a syndrome of diverse neurological manifestations and peripheral blood acanthocytosis. Cases may be sporadic or familial (autosomal dominant or recessive) [4-6]. Involuntary movements are the clinical hallmark. Oro-facio-lingual dyskinesias lead to dysarthria, dysphagia, vocalisations and sometimes self-multilation $[2,4]$. Limb chorea and dystonia are common. Behavioural and personality change, dementia, extrapyramidal features, generalised seizures and an axonal neuropathy with areflexia are characteristic features $[2,4]$. Demonstration of over 3\% acanthocytes in peripheral blood is required for diagnosis $[4,7]$. Requesting a 'blood picture' on clinical suspicion is important, as most laboratories now use automated analysers. Repeated sampling may be necessary, and electron microscopy helps diagnosis [4]. MRI scanning 
may show a high $\mathrm{T} 2$ signal in the basal ganglia and caudate atrophy. Normal plasma lipid levels and lipoprotein electrophoresis help to distinguish this from abetalipoproteinaemia $[4,6,8]$.

Proper evaluation of movement disorders in young people is necessary as there are important considerations regarding diagnosis, treatment and family counselling. Paroxysmal dyskinesias are commonly mistaken for seizures. Chorea and cognitive impairment in neuroacanthocytosis can mimic Huntington disease, with implications for genetic counselling [6,8]. Dyskinesias and dystonias interfere with activities of daily living and occupation, leading to disability and handicap. Appropriate management depends on accurate diagnosis. As shown by these cases, the diagnosis can sometimes be established on clinical grounds or with simple investigations.

\section{References}

1. Munchau A, Valente EM, Shahidi GA, Eunson LH, Hanna $\mathrm{MG}$, et al. A new family with paroxysmal exercise induced dystonia and migraine; a clinical and genetic study. Journal of Neurology, Neurosurgery and Psychiatry 2000;

\section{8: $609-14$}

2. Riley DE, Lang AE. Movement disorders. In: Bradley WG, Daroff RB, Fenichel GM, Marsden CD, eds. Neurology in Clinical Practice. Massachusetts: ButterworthHeinemann, 1996; 1733-72

3. Plant GT, Williams AC, Earl CJ, Marsden CD. Familial paroxysmal dystonia induced by exercise. Journal of Neurology, Neurosurgery and Psychiatry 1984; 47: 275-9.

4. Hardie RJ, Pullon HWH, Harding AE, Owen JS, Pires M, et al. Neuroacanthocytosis- a clinical, haematological and pathological study of 19 cases. Brain 1991; 114: 13-49

5. Aminoff MJ. Acanthocytosis and neurological disease. Brain 1972; 95: 749-60

6. Hardie RJ. Acanthocytosis and neurological impairmenta review. Quarterly Journal of Medicine 1989; 71: 291-306

7. Wills A, Marsden CD, eds. Fifty Neurological Cases from the National Hospital. London: Martin Dunitz, 1999

8. Sotaniemi KA. Chorea-acanthocytosis-Neurological disease with acanthocytosis. Acta neurologica scandinavica 1983; 68: 53-6. 